\title{
$600-1112-169$
}

UM HE 73-2

This report was prepared as an account of work sponsored by the United States Government. Neither the United States nor the United States Atomic Energy Commission, nor any of their employees, nor any of their contractors, subcontractors, or their employees, makes any warranty, express or implied, or assumes any legal liability or responsibility for the accuracy, com pleteness or usefulness of any information, apparatus, product or process disclosed, or represents that its use would not infringe privately owned rights.

\section{Energy Dependence of Mean Square Charged Multiplicity in Regge-Mueller Formalism}

S. Rai Choudhury* and D.P. Majumdar

Randall Laboratory of Physics

University of Michigan

Ann Arbor, Michigan 48104

*on leave from the University of Delhi, Delhi, India. 


\section{DISCLAIMER}

This report was prepared as an account of work sponsored by an agency of the United States Government. Neither the United States Government nor any agency Thereof, nor any of their employees, makes any warranty, express or implied, or assumes any legal liability or responsibility for the accuracy, completeness, or usefulness of any information, apparatus, product, or process disclosed, or represents that its use would not infringe privately owned rights. Reference herein to any specific commercial product, process, or service by trade name, trademark, manufacturer, or otherwise does not necessarily constitute or imply its endorsement, recommendation, or favoring by the United States Government or any agency thereof. The views and opinions of authors expressed herein do not necessarily state or reflect those of the United States Government or any agency thereof. 


\section{DISCLAIMER}

Portions of this document may be illegible in electronic image products. Images are produced from the best available original document. 
The energy dependence of the nean square multiplicity $\left\langle n^{2}\right\rangle$ is obtained on the basis of a regge-Mueluer anal.ysis, when a Pomeron with $\alpha_{p}(0)=1$. and subsidiary trajectories with $\alpha_{p}(0)=I / 2$ are present. The expression for $\langle n(n-1)\rangle$ is shown to be of the form $c_{1}(\ell n s)^{2}+c_{2}(\ell n s)+c_{3}+c_{4} \frac{(\ell n s)^{2}}{\sqrt{s}}$ $+c_{5} \frac{\operatorname{lns}}{\sqrt{s}}+\frac{c_{6}}{\sqrt{s}}$, with the coefficients given in terms, of certat. well-aefined integrals over physically measurable quantities. It is found that $c_{2}$ ana $c_{4}$ must be positive. We discuss ho: the sign of $c_{2}$ can be negative to account for the present experimental data. From a knowledge of a similar expression for the mean multiplicity $\langle n\rangle$, plausible numeriral estimates of the coefficients $c_{1}$ and $c_{2}$ are obtained. These two coefficients alone reproduce reasonably well the $\left\langle n_{c h}\left(n_{c h}-1\right)\right\rangle$ experimental data in $p-p$ collisions up to a constant term. 


\section{Intzoduction}

The recent measurementss ${ }^{2}$ of the noan multiplicj.ty $\left\langle\mathrm{n}_{\mathrm{ch}}\right\rangle$ and the mean square multipli.city $\left\langle\mathrm{n}_{\mathrm{ch}}^{2}\right\rangle$ of charged particles in high energy hadronic collisions pxovide a great deal of information about high energy collisions. These multiplicities can be expressed as integrals over inclusive cross-sections and hence the study of the energy dependence of these multiplicities is a very important means to check our understanding of inclusive cross-sections. Several models have been proposed in the past to study hadronic interactions for infinitely large energies. One cannot claim with certainty that the present experimental observations have already reached this asymptotic region. There are already indications that the sub-asymptotic terms are important all the way up to ISR energies ${ }^{3}$. It is thus very important to know the sub-asymptotic models, so that one can profitably compare the present experimental data with the expectations of a theory. Unfortunately, most of the proposed models are not defined enough in the sub-asymptotic regions because of large freedoms inherent: in the models. In this respect the Regge-Mueller ${ }^{4}$ description of the high energy inclusive reactions is unique in the sense. that it has a definite prescription of calculating the subasymptotics. If the exchanged objects in a Regge-Mueller theory are taken to be simple poles that one is familiar with in the study of two-body reactions, then the asymptotic behavior of an inclusive cross-section is given by the pomeron 
exchange whereas the exchänges of lower Regge poles gjve the sub-asymptotics. It is known that the main fatures of twobody reactions can be reasonably well described by a pomeron with $\alpha_{p}(0)=1$ and four subsidiary trajectories $f_{0^{\prime}} w, p$, and $A_{2}$ all with an intercept of $\alpha_{p^{\prime}}(0)$ approximately $1 / 2$. In the description of inclusive reactions, where only forward helicity non-flip protons are involved, we can neglect, as a good first approximation the isovector trajectories. Calculation of ( $n_{c h}$ ) on the basis of such a Regge-Muellex model has already been done 5 and they seem to accomodate well the present data from lab momentum 4 to $10^{4} \mathrm{GeV} / \mathrm{c}$. It has become clear, however, that the mean square multiplicity $\left\langle n_{c h}^{2}\right\rangle$ rather than $\left\langle n_{c h}\right\rangle$ is more of a distinctive characteristic of hadronic models. It is therefore important to know the energy dependence of $\left\langle n_{c h}^{2}\right\rangle$ in any theoretical model and we intend to discuss this from the Regge-Mueller theory. We conisider pomeron, $w$, and $P^{\prime}$ exchanges, and derive the expression for the leading and the non-leading terms for $\left\langle\mathrm{n}_{\mathrm{ch}}\left(\mathrm{n}_{\mathrm{ch}}-1\right)\right\rangle$.

In section II we define the relevant couplings and write down the cross-sections in different regions of the phasespace. The mean multiplicities $\left\langle n_{c h}\right\rangle$ and $\left\langle n_{c h}^{2}\right\rangle$ are then calculated keeping all the terms in this theory. The coefficients of these expansions are discussed in the next section and it is shown that reasonable fits of the data for large s are possible with our estimates of the leading terms. 
II. Behavior of $\langle n\rangle$ and $\left\langle n^{2}\right\rangle$.

We consider for definiteness collisions of two protons each of mass $m$. We work throughout in the c.m. system so that the two incoming momenta are $p_{a} \equiv m(\cosh \theta, 0,0,-\sinh \theta)$ and $\mathrm{p}_{\mathrm{b}} \equiv \mathrm{m}(\cosh \theta, 0,0, \cdot \sinh \theta)$. Assuming exchanges of pomeron with $\alpha_{p}(0) \equiv \alpha_{p}=1$ and $f_{0}$ and w with $\alpha_{i}(0) \equiv \sigma_{i}=1 / 2, i=w, f_{0}$ the total cross-section has the expansion

$$
\sigma_{t}=\frac{1}{s}\left[e^{2 \theta} \gamma_{p}^{2}+\sum_{i} e^{\theta} \gamma_{i}^{2}\right]
$$

$\gamma_{i}$ 's are the Regge-particle couplings; in Eq(I) and in equations to follow all summations run over $f_{0}$ and $w$. Consider now the one-particle inclusive reaction

$$
p+p \rightarrow \pi^{-}+\text {Anything, }
$$

for a $\pi^{-}$of momenta $p_{1} \equiv \mu\left(\cosh \theta_{1} \cosh \beta_{1} \cdot \sinh \beta_{1}, 0, \sinh \theta_{1} \cosh \beta_{1}\right)$, where $\mu$ is the pion mass. $\beta_{1}$ ranges from 0 to $\infty$, whereas $\theta_{1}$ varies between $\pm \theta_{m}$ where $\theta_{m}=\theta+\ln \left(m / \mu_{t}\right), \mu_{t}$ being the transverse mass of the pion. For the fragmentation region where $\left(\theta+\theta_{1}\right)$ is less than a certain number $\theta_{0}$ say, we have the expansion ${ }^{6}$.

$\frac{d \sigma}{d \theta_{1} d\left(\cosh \beta_{1}^{2}\right)}=\frac{l}{s}\left[e^{2 \theta} f_{p}\left(\theta_{1}+\theta, \beta_{1}\right) \gamma_{p}+\sum_{i} e^{\theta} f_{i}\left(\theta_{1}+\theta, \beta_{1}\right) \gamma_{i}\right] ;$ an exactly similar expression holds at the other end where $\left(\theta-\theta_{1}\right)$ is less than $\theta_{0}$. The $f_{i}$ 's are the usual couplings of the Regge pole $i$ to the ( $p \pi)$ system. In the pionization region where $\theta$ lies between $\left(-\theta+\theta_{0}\right)$ and $\left(\theta-\theta_{0}\right)$, the inclusive cross-section is given by 


$$
\begin{aligned}
\frac{d \sigma}{d \theta_{1} d\left(\operatorname{coshn} \beta_{1}^{2}\right)}= & \frac{1}{s}\left[e^{20} f_{p p}\left(\beta_{1}\right) \gamma_{p}^{2}\right. \\
& +2 \exp \left(\frac{3}{2} \theta\right) \cosh \left(1 / 2 \theta_{1}\right) \sum_{i} f_{i p}\left(\beta_{1}\right) \gamma_{p} \gamma_{i} \\
& \left.+\exp (\theta) \sum_{i} f_{i i}\left(\beta_{1}\right) \gamma_{i}^{2}\right] .
\end{aligned}
$$

The $f_{i} i$, are the four point coupling of Regge-poles $i$, $i$ ' to a pion line. We defer a discussion of the actual value of $\theta_{0}$ until later in this section.

Consider now the two-particle inclusive cross-section.

$$
p+p \rightarrow \pi^{-}\left(p_{1}\right)+\pi^{-}\left(p_{2}\right)+\text { Anything }
$$

where the momenta $p_{1}$ is defined as before and $p_{2}=\mu\left(\cosh \theta_{2}\right.$ $\left.\cosh \beta_{2}, \sinh \beta_{2} \cos \varphi_{2}, \sinh \beta_{2} \sin n_{2}, \sinh \beta_{2} \cosh \beta_{2}\right)$. We divide the entire phase space into the following regions and write down the expansions appropriate to each region. We restrict ourselves to $\theta_{2}>\theta_{1}$; an identical set of expansions hold for the complementary region $\theta_{2}<\theta_{1}$.

(i) Simple fragmentation region characterized by $\theta_{2}<-\theta+\theta_{0}$ : In this region we have.

$$
\begin{aligned}
& \frac{d \sigma}{d \theta_{1} d\left(\cosh \beta_{1}{ }^{2}\right) d \theta_{2} \alpha\left(\cosh \beta_{2}{ }^{2}\right) d \varphi_{2}}=\frac{1}{s}\left[e^{2 \theta_{D}} D_{p}\left(\theta_{1}+\theta_{1} \theta_{2}-\theta_{1}, \beta_{1}, \beta_{2}, \mathrm{CO}_{2}\right)\right. \\
& \left.+\sum_{i} e^{\theta} D_{i}\left(\theta_{1}+\theta, \theta_{2}-\theta_{1}, \beta_{1}, \beta_{2}, \varphi_{2}\right)\right]
\end{aligned}
$$

$D_{i}$ 's represent the coupling of the Regge pole $i$ to the (p $\pi \pi$ ! system.

(ii) Double fragmentation region characterized by $\theta_{1}<-\theta+\theta_{0}$ and $\theta_{2}>\theta-\theta_{0}$ : The inclusive cross-section in this region is given by 


$$
\begin{aligned}
\frac{d \sigma}{d \theta_{1} d\left(\cosh \beta_{1}{ }^{2}\right) d \theta_{2} d\left(\cosh \beta_{1}{ }^{2}\right) d \theta_{2}} & =\frac{1}{s}\left[e^{2 \theta_{f_{p}}}\left(\theta_{1}+\theta_{1} \beta_{1}\right) f_{p}\left(\theta-\theta_{2}, \beta_{2}\right) \frac{1}{2 \pi}\right. \\
& \left.+\sum_{i} e^{\theta_{1}} f_{i}\left(\theta_{1}+\theta_{1} \beta_{1}\right) f_{i}\left(\theta-\theta_{2}, \beta_{2}\right) \frac{1}{2 \pi}\right] ; \quad \text { (7) }
\end{aligned}
$$

The $f_{i}$ 's are the same ones occurring in Eq (3) so that we are building in factorization right from the beginning.

(iii) Pionization-Fragmentation region characterized by $-\theta<$

$\theta_{1}<-\theta+\theta_{0}$ and $-\theta+\theta_{0}<\theta_{2}<\theta-\theta_{0}$ : The cross-section in this domain is given by

$$
\begin{aligned}
& \frac{d \sigma}{d \theta_{1} d\left(\cosh \beta_{1}{ }^{2}\right) d \theta_{2} d\left(\cosh \beta_{2}{ }^{2}\right) d \theta_{2}}=\frac{1}{s} \cdot \frac{1}{2 \pi}\left[\exp (2 \theta) \gamma_{p} f_{p}\left(\theta_{1}+\theta, \beta_{1}\right) f_{p p}\left(\beta_{2}\right)\right. \\
& \quad+\exp \left(\frac{3 \theta+\theta_{2}}{2}\right) \sum_{i} \gamma_{i} f_{p}\left(\theta_{1}+\theta, \beta_{1}\right) f_{p i}\left(\beta_{2}\right) \\
& +\exp \left(\frac{3 \theta-\theta_{2}}{2}\right) \sum_{i} \gamma_{p} f_{i}\left(\theta_{1}+\theta, \beta_{1}\right) f_{p i}\left(\beta_{2}\right) \\
& \left.+\exp (\theta) \sum_{i} \gamma_{i} f_{i}\left(\theta_{1}+\theta, \beta_{1}\right) f_{i i}\left(\beta_{2}\right)\right] .
\end{aligned}
$$

We next come to the region where both the pions are emitted in the central region. We further subdivide this into two domains depending on whether $\left(\theta_{2}-\theta_{1}\right)$ is bigger than or less than a certain fixed number 8 . The cross-sections in these two regions have the following form.

(iv) Simple pionization region characterized by $-\theta+\theta_{0}<\theta_{1}<$ $\theta_{2}<\theta-\theta_{\mathrm{o}}$ and $\theta_{2}-\theta_{1}<\delta$ : In this region $\frac{d \sigma}{d \theta_{1} \alpha\left(\cosh \beta_{2}{ }^{2}\right) \alpha \theta_{2}\left(\cosh \beta_{2}{ }^{2}\right) d \theta_{2}}=\frac{l}{s}$

$$
\left[\exp (2 \theta) \exp \left(\theta_{1}-\theta_{2}\right) g_{p p}\left(\theta_{2}-\theta_{1}, \beta_{1}, \beta_{2}, \theta_{2}\right) \gamma_{p}^{2}\right.
$$


$-6-$

$$
\begin{aligned}
& +\exp \left(\frac{3}{2} \theta\right) \exp \left(\theta_{1}-\theta_{2} / 2\right) \sum_{i} g_{i p}\left(\theta_{2}-\theta_{1}, \beta_{1}, \beta_{2}, \omega_{2}\right) \gamma_{p} \gamma_{i} \\
& +\exp \left(\frac{3}{2} \theta\right) \exp \left(\theta_{1} / 2-\theta_{2}\right) \sum_{i} g_{i p}\left(\theta_{2}-\theta_{1}, \beta_{1}, \beta_{2}, \omega_{2}\right) \gamma_{p} \gamma_{i} \\
& \left.+\exp (\theta) \exp \left(\theta_{1} / 2 \cdot-\theta_{2} / 2\right) \sum_{i} g_{i i}\left(\theta_{2}-\theta_{1}, \beta_{1}, \beta_{2}, \theta_{2}\right) \gamma_{i}{ }^{2}\right]
\end{aligned}
$$

The $g_{i i}$, represent the six-point coupling of the Reggae poles

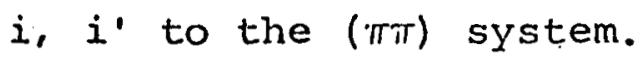

(v) Double pionization region characterized by $-\theta+\theta_{0}<\theta_{1}<\theta_{2}<$ $\theta-\theta_{0}$ and $\theta_{2}-\theta_{1}>8$. The expansion for this region is

$$
\begin{aligned}
& \frac{d \sigma}{d \theta_{1} d\left(\cosh \beta_{1}{ }^{2}\right) d \theta_{2}\left(\cosh \beta_{2}{ }^{2}\right) d \theta_{2}}=\frac{1}{s} \cdot \frac{1}{2 \pi} \\
& \quad\left[\exp (2 \theta) \gamma_{p}{ }^{2} f_{p p}\left(\beta_{1}\right) f_{p p}\left(\beta_{2}\right)\right. \\
& +\exp \left(2 \theta+\frac{\theta_{1}}{2}-\frac{\theta_{2}}{2}\right) \sum_{i} \gamma_{p}^{2} f_{i p}\left(\beta_{1}\right) f_{i p}\left(\beta_{2}\right) \\
& +\exp \left(\frac{3 \theta}{2}+\frac{\theta_{2}}{2}\right) \sum_{i} \gamma_{p} \sum_{i} f_{p p}\left(\beta_{1}\right) f_{i p}\left(\beta_{2}\right) \\
& +\exp \left(\frac{3 \theta}{2}+\frac{\theta_{1}}{2}\right) \sum_{i} \gamma_{p} \gamma_{i} f_{p i}\left(\beta_{1}\right) f_{i i}\left(\beta_{2}\right) \\
& +\exp \left(\frac{3 \theta}{2}-\frac{\theta_{I}}{2}\right) \sum_{i} \gamma_{p} \gamma_{i} f_{i p}\left(\beta_{1}\right) f_{p p}\left(\beta_{2}\right) \\
& +\exp \left(\theta+\theta_{2}-\theta_{1}\right) \sum_{i} \gamma_{i} f_{i p}\left(\beta_{1}\right) f_{i p}\left(\beta_{2}\right) \\
& +\exp \left(\frac{3 \theta}{2}-\theta_{2}\right) \sum_{i} \gamma_{p} \gamma_{i} f_{i p}\left(\beta_{1}\right) f_{p p}\left(\beta_{2}\right) \\
& \left.+\frac{\theta_{2}}{2}\right) \sum_{i} \gamma_{p} \gamma_{i} f_{i i}\left(\beta_{1}\right) f_{p i}\left(\beta_{2}\right) \\
& +
\end{aligned}
$$




$$
\left.+\exp \left(\theta+\frac{1}{2} \theta_{2}-\frac{l}{2} \theta_{1}\right) \sum_{i} \gamma_{j}^{2} f_{i i}\left(\beta_{1}\right) f_{i . i}\left(\beta_{2}\right)\right]
$$

The mean and mean square multiplicities are related to inclusive cross-section by the relations:

$$
\sigma_{t}\left\langle n_{-}\right\rangle=\int_{1}^{\infty} d\left(\cosh \beta_{1}^{2}\right) \int_{-\theta_{m}}^{\theta_{m}} d \theta_{1} \frac{d \sigma}{d \theta_{1} d\left(\cosh \beta_{1}^{2}\right)}
$$

and

$$
\begin{aligned}
\sigma_{t}\left\langle n_{-}\left(n_{-}-1\right)\right\rangle= & \int_{0}^{2 \pi} d \omega_{2} \int_{1}^{\infty} d\left(\cosh \beta_{1}{ }^{2}\right) \int_{1}^{\infty} d\left(\cosh \beta_{2}{ }^{2}\right) \\
& x \int_{-\theta_{m 1}}^{\theta_{m 1}} d \theta_{1} \int_{-\theta_{m 2}}^{+\theta_{m 2}} d \theta_{2} \frac{d \sigma}{d \theta_{1} d\left(\cosh \beta_{1}{ }^{2}\right) d \theta_{2} d\left(\cosh \beta_{2}{ }^{2}\right) d \omega_{2}}
\end{aligned}
$$

where $\theta_{m i}=\theta+\ln \frac{m}{\mu \cosh \beta_{i}}, i=1,2$. Using the expressions in Eqs (6) through (10), we obtain the following expansion for the mean multiplicity ${ }^{5}$ :

$$
\begin{aligned}
\sigma_{t}\left\langle n_{-}\right\rangle= & F_{p p} \gamma_{p}^{2} l n s+2\left[\gamma_{p} F_{p}-\theta_{o} F_{p p} \gamma_{p}^{2}+2 \sum_{i} F_{p i} \gamma_{p} \gamma_{i} \exp \left(-\frac{1}{2} \theta_{0}\right)\right] \\
& +\sum_{i} F_{i i} \gamma_{i}^{2} \ell n s / \sqrt{s} \\
& +\frac{2}{\sqrt{s}}\left[\sum_{i} \gamma_{i} F_{i}-\theta_{0} \sum_{i} F_{i i} \gamma_{i}^{2}-2 \sum_{i} F_{p i} \gamma_{p} \gamma_{i} \exp \left(1 / 2 \theta_{0}\right)\right]:
\end{aligned}
$$

\section{where}

$$
F_{i}=\int_{i}^{\infty} d\left(\cosh \beta_{1}^{2}\right) \int_{-\theta_{m l}}^{-\theta_{m l}+\theta_{0}} d \theta_{1} F_{i}\left(\theta+\theta_{1}, \beta_{1}\right) ; i=p, f_{0^{\prime}} w
$$




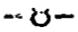

and

$$
F_{i i^{\prime \prime}}=\int_{1}^{\infty} d\left(\cosh \beta_{1}^{2}\right) f_{i i^{\prime}}\left(\beta_{1}\right) ; i, \ddot{i}^{\prime}=p, f_{0^{\prime}}, w .
$$

The functions $F_{i}$ and $F_{i i}$, are independent of energy and are functions of $\theta_{0}$ only. Further, since $w$ has negative G-parity

$$
\begin{aligned}
F_{p w}=F_{f_{0} w}=0 . & \text { For }\left\langle n_{-}\left(n_{-}-1\right)\right\rangle \text { we obtain } \\
\sigma_{t}\left\langle n_{-}\left(n_{-}-1\right)\right\rangle= & \sigma_{t}\left[c_{1}(\ell n s)^{2}+c_{2}(2 n s)+c_{3}+c_{4} \frac{(\text { Ins })^{2}}{\sqrt{s}}\right. \\
& \left.+c_{5} \frac{1, n s}{\sqrt{s}}+\frac{c_{6}}{\sqrt{s}}\right]
\end{aligned}
$$

where $c_{i}$ 's are given as follows:

$$
\begin{aligned}
& \sigma_{t} c_{1}=g_{p p}^{p} \\
& \sigma_{t} c_{2}=-4\left(\theta_{0}+\delta\right) g_{p p}^{p}+\sum_{i} 4 g_{p p}^{i} \exp (-\delta)+\sum_{i} 8 g_{i p}^{p} \exp \left(-\theta_{0} / 2\right) \\
& +2 \gamma_{p}^{2} h_{p p}^{3}+4 \gamma_{p} F_{p} F_{p p} \\
& \sigma_{t} c_{3}=4 g_{p p}^{p}\left(\theta_{0}+\delta\right)^{2}-\sum_{i} 8 g_{p p}^{i}\left(\theta_{0}+\delta+1\right) \exp (-\delta) \\
& +8 \sum_{i} g_{i i}^{p} \exp \left(-\theta_{0}\right)-\sum_{i} 16 g_{i p}^{p}\left(\theta_{0}+\delta+1\right) \exp \left(-\theta_{0} / 2\right) \\
& +16 \exp \left(-\theta_{0} / 2-\delta\right) \sum_{i} g_{i p}^{i}-4 \theta_{o} \gamma_{p}^{2} h_{p p}^{(3)} \\
& -2 \gamma_{p}^{2} h_{p p}^{(1)}+8 \sum_{i} g_{i p}^{(3)} \gamma_{p} \gamma_{p^{\prime}} \exp \left(-\theta_{0} / 2\right) \\
& -8 \theta_{\circ} \gamma_{p} F_{p} F_{p p}+8 \exp \left(-\theta_{0} / 2\right) \sum_{i} \gamma_{i} F_{p} F_{p i} \\
& +8 \exp \left(-\theta_{0} / 2\right) \sum_{i} \gamma_{p} F_{i} F_{p i}+2 \gamma_{p} D_{p}+2 F_{p}{ }^{2} \text {, }
\end{aligned}
$$




$$
\begin{aligned}
\sigma_{t} c_{4}= & \sum_{i} g_{i i}^{j}, \\
\sigma_{t} c_{5}^{i}= & -4\left(\theta_{0}+\delta\right) \sum_{i} g_{i i}^{i}-4 \exp (\delta) \sum_{i} g_{i i}^{p}-8 \exp \left(\theta_{0} / 2\right) \sum_{i}^{p} g_{i p}^{i} \\
& +2 \sum_{i} \gamma_{i}^{2} h_{i i}^{(2)}+4 \sum_{i} \gamma_{i} F_{i} F_{i i},
\end{aligned}
$$

and

$$
\begin{aligned}
& \sigma_{t} c_{6}=8 \sum_{i} g_{p p}^{i} \exp \left(\theta_{0}\right)+4\left(\theta_{0}+\delta\right)^{2} \sum_{i} g_{i i}^{i} \\
& +8\left(\theta_{0}+\delta-1\right) \exp (\delta) \sum_{i} q_{i i}^{p} \\
& +16\left(\theta_{0}+\delta-1\right) \exp \left(\theta_{0} / 2\right) \sum_{i} g_{i p}^{i} \\
& +16 \exp \left(\theta_{0} / 2+\delta\right) \sum_{i} g_{i p}^{p}-4 \theta_{0} \sum_{i} \gamma_{i}^{2} h_{i i}^{(2)} \\
& -2 \sum_{i} \gamma_{i}^{2}{ }^{n}{ }_{i i}^{(4)}-8 \exp \left(\theta_{0} / 2\right) \sum_{i} \gamma_{p} \gamma_{i} n_{i p}^{(2)} \\
& -8 \theta_{0} \sum_{i} \gamma_{i} F_{i} F_{i i}-8 \exp \left(\theta_{0} / 2\right) \sum_{i} \gamma_{i} F_{p} F_{p i} \\
& -8 \exp \left(\theta_{0} / 2\right) \sum_{i} \gamma_{p} F_{i} F_{p i}+2 \sum_{i} \gamma_{i} D_{i}+2 \sum_{i} F_{i}^{2} .
\end{aligned}
$$

We have defined here

$$
g_{i \cdot i "}^{i}=\gamma_{i} \cdot \gamma_{i \prime \prime} F_{i i} \cdot F_{i i^{\prime \prime}}
$$

The $h_{i j}^{(I)}$ etc. are given by:

$$
\begin{aligned}
h_{i i}^{(\lambda)} & \equiv \int_{0}^{2 \pi} d \varphi_{2} \int_{1}^{\infty} d\left(\cosh \beta_{1}{ }^{2}\right) \int_{1}^{\infty} d\left(\cosh \beta_{2}{ }^{2}\right) \int_{0}^{\delta} d \theta_{21} h(\lambda)\left(\theta_{21}\right) \\
& \times g_{i i}\left(\theta_{21}, \beta_{1}, \beta_{2}, \varphi_{2}\right), \quad(\lambda=1, \ldots 4),
\end{aligned}
$$


with $h^{(1:}(x)=x \exp (-x), h^{(2)}(x)=\exp (-x / 2), h^{(3)}(x)=\exp (-x)$, and $h^{(4)}(x)=x \exp (-x / 2)$; The D's are

$$
\begin{aligned}
D_{i}= & \int_{0}^{2 \pi} d \varphi_{2} \int_{1}^{\infty} d\left(\cosh \beta_{1}^{2}\right) \int_{1}^{\infty} d\left(\cosh \beta_{2}{ }^{2}\right) \cdot \int_{-\theta_{m 1}}^{-\theta_{1}+\theta_{0}} d \theta_{1} \int_{-\theta_{m 2}}^{-\theta_{m 2}+\theta_{0}} d \theta_{2} \\
& \times D_{i}\left(\theta_{1}+\theta, \theta_{2}-\theta_{1}, \beta_{1}, \beta_{2}, \varphi_{2}\right) .
\end{aligned}
$$

We conclude this section with the following two observations: (1) To be technically correct, we should use the expansion in $E q(1)$ for $\sigma_{t}$ to obtain the final expansion for $\left\langle n_{-}\right\rangle$and $\left\langle n_{-}\left(n_{-}-1\right)\right\rangle$. However, for all practical purposes $\sigma_{t}$ is a remarkably constant quantity from the accelerator energies to ISR energies, so that in studying the energy dependence of $\left\langle n_{-}\right\rangle$and $\left\langle n_{-}\left(n_{-}-l\right)\right\rangle$ via Eqs(13) and (IG) it is reasonable to assurne $\sigma_{t}$ to be constant.

(2) The quantity $\theta_{0}$ we have introduced divides the phase space into fragmentation and pionization domains. It is trivially true that if the expansion used in various regions matches at the boundaries, the expressions for $\left\langle n_{-}\right\rangle$and $\left\langle n_{-}\left(n_{-}-1\right)\right\rangle$ will not be sensitive to $\theta_{0}$ as $\theta_{0}$ is varied in the pionization-fragmentation boundary region. To obtain a reasonable value of $\theta_{0}$ consider the subenergy in the one-particle inclusive reaction given by

$$
\left(p_{0}+p_{1}\right)^{2}=m^{2}+\mu^{2}+2 \mu_{t} \exp \left(\theta+\theta_{1}\right)
$$

From our experience in analysis of two-body scattering, we can now expect that if the sub-energy (26) is greater than say 
$5(\mathrm{GeV}) \%$, it can be consiciered asymptotic. In other words. the expansions we have used in the pionization region with $P, f_{0}$, w exchanges, would be reasonable if the sub-energy (26) is greater than say about $5(\mathrm{GeV})^{2}$. The boundary of the pionization region is $\theta_{1} \ddot{=}-\theta+\theta_{0}$, so that we obtain an estimate for $\theta_{0} \approx 1.7$. In a similar way, the parameter $\delta$ divides the entire pionization into simple and double pionization regions depending on the sub-energy of the two pions. Following an analogous argument as used above for $\theta_{0}$ we can estimate a value for $\delta$ which would not be very aifferent from that of $\theta_{0}$;

III. Numerical Evaluation and Discussion

We have seen that the coefficients in the expansion for $\left\langle n_{-}\right\rangle$and $\left\langle n_{-}\left(n_{-}-1\right)\right\rangle$ can be evaluated once we know certain Regge-particle couplings defined in the previous saction. We shall now discuss possible numerical estimates for these coefficients. We note at the very beginning that precise predictions for these coefficients are not possible at present, due mainly to our lack of exact knowledge of the couplings of the subsidiary trajectories in inclusive cross-sections.

First, the best fit to the total cross-section data tells us that ${ }^{7}$

$$
\gamma_{p}^{2}=37.4
$$

Further, in the approximation of neglecting couplings of $\rho$ and $A_{2}$ to the forward helicity non-flip amplitude, we have from Ref. 7 


$$
\gamma_{\mathrm{f}}^{2}=\text { coefficient of } l / \sqrt{\mathrm{s}} \text { term in }\left(\sigma_{\mathrm{pp}}+\sigma_{\bar{p} p}\right) / 2=42 \text {. }
$$

Next, Tow has carried out a. fit for $\left\langle n_{c h}\right\rangle$ of the form

$$
\left\langle\mathrm{n}_{\mathrm{ch}}\right\rangle=\mathrm{A}+\mathrm{blns}+\mathrm{d} \mathrm{s}^{-l / 2} \text {, }
$$

and the values for the best fit are $a=-4.8, b=2.0$, and $d=10.0$. This fit is comparable to the fits obtained by other people $e^{14}$ and we shall use it only to obtain some rough estimates. Assuming $n_{c h}=2 n_{-}+2$, we then immediately obtain

$$
F_{p p} \cong 1.0
$$

which tells us that $c_{1}=F_{p p} 2 \cong 1.0$.

Now the fit (29) does not distinguish between $1 / \sqrt{ } s$ and ens/ $\sqrt{\mathrm{s}}$ term, so that in comparing (29) with (13), we will ieplace ins/ $\sqrt{5}$ in (13) zy an effective average valüe ( $n s$ ) av/Js. Assuming that, we have

$$
\begin{aligned}
10.0 \cong & \frac{2}{\sigma_{t}}\left[\sum_{i}\left\{(\ell n s)_{a v .}-2 \theta_{0}\right\} F_{i i} \gamma_{i}^{2}-4 \exp \left(\theta_{0} / 2\right) F_{p E} \gamma_{p} \gamma_{f}\right. \\
& \left.+\sum_{i} 2 \gamma_{i} F_{i}\right] .
\end{aligned}
$$

For large $\theta_{0}$, the R.H.S. of (3I) will be dominated by the second term. For our choice of $\theta_{0}$, we will retain as a first approximation only the second term; for $\theta_{0}=2$, this gives

$$
F_{p E} \cong-0.43 \text {. }
$$

The -ve sign of $F_{p f}$ is evident from the fact that the pionization cross-section rises with energy from the accelerator to ISR energies. The fragmentation integral $F_{p}$ can be easily estimated, because it is known that fragmentation reaches a limiting behavior rather early and at accelerator energies 
reasonably detailed information is available ${ }^{8}$ for us to astimate the integral $F_{p}$ in $E q(24)$. For $\theta_{0}=2$, our estimate is

$$
F_{p} \simeq 2.0
$$

At this stage, we are unable to make numerical estimates of the other quantities needed to determine all the $c_{i}$ 's. However, by using duality arguments, we can expect that in the pionization region, the double pionization formula given in Eq (10) continues to describe the simple pionization region IV in an average sense. This means that a reasonable estimate for $c_{2}$ can be made by putting $\delta=0$ and $h_{p p}^{3}=0$ in $E q(18)$. Numerically this means

$$
c_{2} \approx-8+0.74-1.34+1.31=-7.29 \text {. }
$$

Because of our lack of knowleage of the $c_{i}$ 's beyond $c_{2}$ ' the best we can do at this stage in comparing experjmental data on $\left\langle n_{\mathrm{ch}}\left(\mathrm{n}_{\mathrm{ch}}-1\right)\right\rangle$ with the Regge-Mueller model, is to attempt a fit to the data retaining the first three terms in Eq(16) (which are the only ones that survive anymptotically), using the determined values of $c_{1}$ and $c_{2}$ and treating $c_{3}$ as a parameter. To obtain $\left\langle n_{\mathrm{ch}}\left(\mathrm{n}_{\mathrm{ch}}-1\right)\right\rangle$ from our results we use the expression $4\left\langle n_{-}\left(n_{-}-1\right)\right\rangle+5\left\langle n_{c h}\right\rangle-8$ for it. Figure 1 shows the fit obtained for $\theta_{0}=2.0$. We also show in the figure the results for $\theta_{0}=1.0$. The two curves show some difference, the reason being that in our approximate estimates for the various couplings, the fragmentation and pionization crosssection have failed to merge into each other smoothly. Because of the uncertainties in determining the Regge parameters, and ovx inabijity to make numerical estjmates for the coeffj.- 
cients $c_{3}$ to $c_{6}$, we cannot make too stiong a conclusion firom our fits. All that we can say is that the experimental data seem to be consistent with our model and the negative sign of. $c_{2}$ which is a marked feature of the data, comes out reasonably well in our model.

Let us now see what more we can learn about the sign of the ins coefficient. We note that only three regions of the phase space contribute to this coefficient. Out of these three the contributions coming from the pionization-fragmentation and the simple pionization regions (via Pomeron exchange) give positive contributions to this coefficient. our rough evaluation of the fragmentation integral tells us that this gives a small contribution, $4 F_{p} / \gamma_{p}$, of the order of 1. The negative sign can then only arise from the Double-Pionization contributions which depend on three factors $\theta_{0}, \delta$, and $F_{p f}$. We have some ideas about the possible ranges of these parameters. We have plotted in Figure 2 the contributions of the double-pionization region to $c_{2}$ ' denoted by $c_{2}$ D.P. as a function of $F_{p f}$ for several reasonable values of $\theta_{0}$ and 8 , namely from 0 to 2. A clear picture is indicated in this graph. If $F_{p f}$ has a small negative value, say between 0 and -1.5 , then $c_{2}$. P. is negative and reasonably large to produce a negative $\ell n s$ coefficient in $\langle n(n-1)\rangle$. This should be true for all reasonable $\theta_{0}$ and $\delta$. We further find that if $\delta=0$ is a good approximation, i.e. the simple pionization region can be approxjmated by the double pionjzation region, then figure 2 shows that $F_{p f}$ must be between -2 and 1. we corount for the negative $c_{2}$. Thus the sign of $\mathrm{c}_{2}$ is 
cruito delicately balanced by $\theta^{\prime}$ onset of the pionization regica, 8 the limit of simple pionization in 2 particle inclusive reactions and the strength of the coupling $F_{p f}$.

It is worthwhile at this point to mention the results that have been derived for other models. In multiperipheral models ${ }^{10}$, one obtains the $(\ell n s)^{2}$ leading term for $\langle n(n-1)\rangle$, but so far one has no prediction for the succeeding terms. In simple fragmentation models ${ }^{11}$ one finds the cross-section for $n$ charged particle production $\dot{\sigma}_{n} \propto 1 / n^{2}$, from which all moments can be calculated. Experimental data ${ }^{l}$ disagree with this simple prediction. A modification of this simple fragmentation model has been make by Hwa ${ }^{12}$ and by Abarbanel and Kane ${ }^{23}$ by taking into account the effect of the minimum momentum fransfer. These latter results can be made to agree with data $^{14}$ by cextain choices of parameters in the model but then the elegance of the model is somewhat lost. These fragmentation models are again aymptotic models and no estimates are available as to how the asymptotic values of the various observables are approached. As we have stated before, our work shows that once some more information on the inclusive cross-sections is available, we will be able to predict all the sub-asymptotic terms and a clear confrontation with experimental data on both $\langle n\rangle$ and $\langle n(n-1)\rangle$ can be made in this theory. 


\section{References and Footnotes}

1. For 7.compilation see S.N. Ganguli and P.K. Malhotra, Phys. Lett. 42B, 83 and 88 (1972). In addition, we have used the following data: J.W. Chapinan, et al., Phys. Rev. Lett. 29, 1686 (1972); F.T. Dao; et al., Phys. Rev. Lett 29,1627 (1972).

2. For a list of references on models see W.R. Frazer, et al., Rev. Mod. Phys. 44, 284 (1971).

3. See for example T. Ferbel, Phys. Rev. Lett. $\underline{29}, 448$ (1972).

4. A.H. Muellex, Phys.: Rev. D2, 2945 (1970):

5. D.M. Tow, SLAC preprint PUB-1110 (1972); R. Cahn (to be published).

6. The expansions used in the various regions for one and two-particle cross-sections are essentially the ones given by H.D.I. Abarbanel, Phys. Rev. D3, 2227 (1971)。

7. V. Barger and R. Phillips, Nucl. Phys. 32B, 93 (1971).

8. W.H. Sims, et al., Vanderbilt University preprint (1972).

9. L. Ratner, et al., Phys. Rev. Lett. 27, 68 (1971)

10. E. Berger, et al., Phys, Rev. Lett. 29, 675 (1972). A.H. Muelier, Phys. Rev. D4, 150 (1971).

11. R. Hwa, Phys. Rev. Lett. 26, 1143 (1972).

12. R. Hwa, Phys. Lett. 42B, 79 (1972).

13. H. Abarbanel and G. Kane, NAL-THY-84 (September 1972).

14. E. Berger, Phys. Rev. Lett. 29 , 887 (1972). 


\section{Figure Captions}

Fig. 1. The available experimental data for $\left\langle\mathrm{n}_{\mathrm{Ch}}\left(\mathrm{n}_{\mathrm{Ch}}{ }^{-1}\right)\right\rangle$ and the predictions. for large $s$ with the coefficients estimated in section 3 are plotted as a function of $\dot{s}$. The solid curve is for $\theta_{0}=2$ and is given by $55+4(\ell n s)^{2}-29 \ell n s+5\left\langle n_{c h}\right\rangle$. The dashed curve is for $\theta_{0}=1$ and is given by $18+$ $4(\ell n s)^{2}-21 \ell n s+5\left\langle n_{c h}\right\rangle$. The constant term in both cases is adjusted for the fit.

Fig. 2. Contributions of the Double-pionization regions to the coefficient of the $\mathrm{lns}$ term in $\left\langle\mathrm{n}_{-}\left(\mathrm{n}_{-}-1\right)\right\rangle$, $\mathrm{C}_{2}{ }^{\text {D.P. }}$, are plotted as a function of $F_{p f}$ for different values of $\theta_{0}$ and 8 . We have set $F_{p p}=1$ and used the value of $\gamma_{f} / \gamma_{p}$ as given by Eqs (28) and (29). 


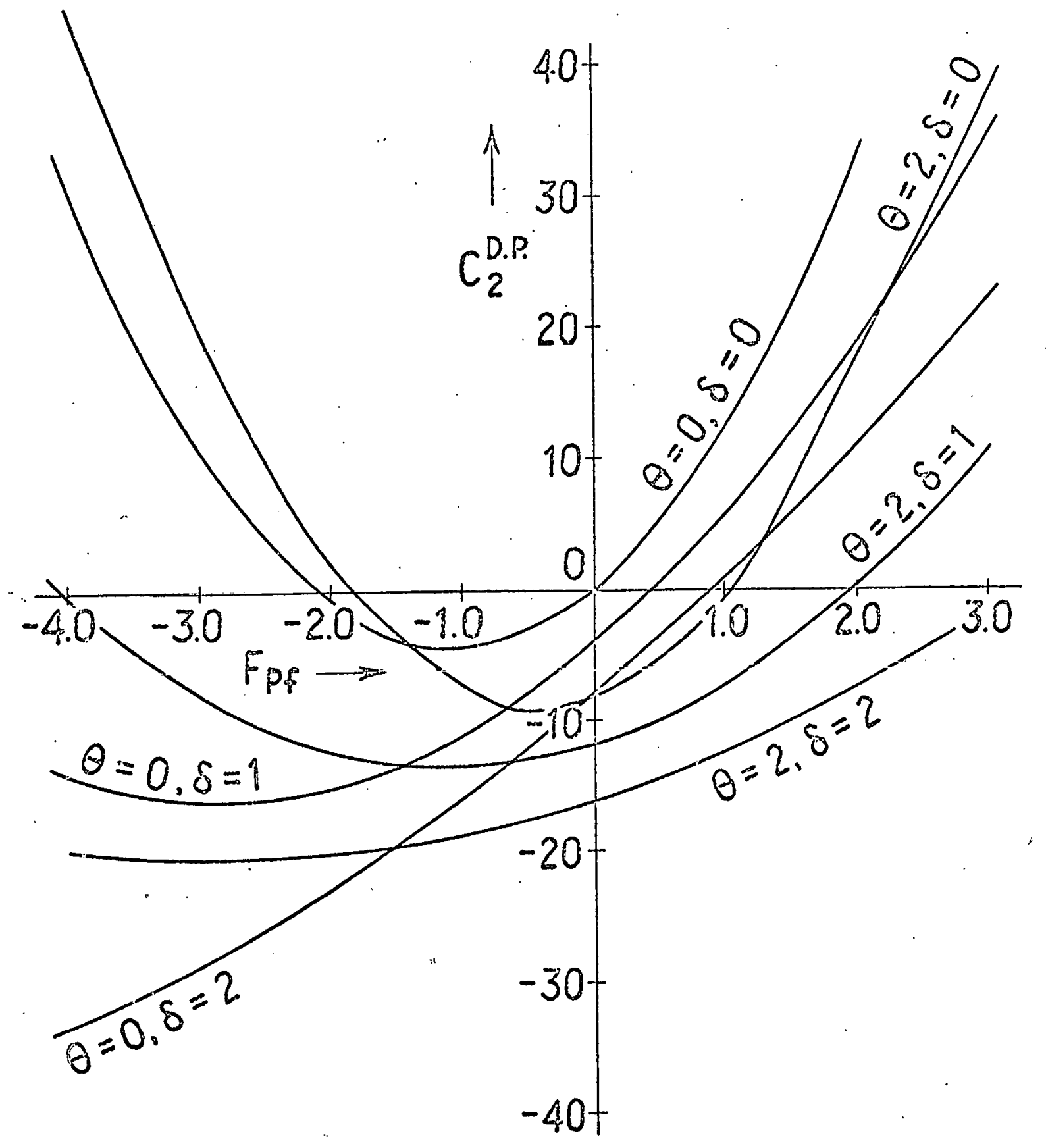

$F_{16} 2$ 